\title{
QUADERNI Quaderni
}

Communication, technologies, pouvoir

70 | Automne 2009

Think tanks, experts et pouvoirs

\section{Le positionnement des think tanks dans l'espace politique américain : des lieux de recherche ou d'influence?}

\section{Cynthia Ghorra-Gobin}

\section{(2) OpenEdition \\ Journals \\ Édition électronique \\ URL : https://journals.openedition.org/quaderni/509 \\ DOI : $10.4000 /$ quaderni.509 \\ ISSN : 2105-2956 \\ Éditeur \\ Les éditions de la Maison des sciences de l'Homme}

Édition imprimée

Date de publication : 5 octobre 2009

Pagination : 57-67

Référence électronique

Cynthia Ghorra-Gobin, « Le positionnement des think tanks dans l'espace politique américain : des lieux de recherche ou d'influence? ", Quaderni [En ligne], 70 | Automne 2009, mis en ligne le 05 octobre 2011, consulté le 28 juin 2022. URL : http://journals.openedition.org/quaderni/509 ; DOI : https:// doi.org/10.4000/quaderni.509 


\section{$D$ ossier}

\section{le positionnement} des think tanks dans l'espace politique américain : des lieux de recherche ou d'influence?

\section{Cynthia Ghorra-Gobin}

Directeur de recherche au CNRS
Les think tanks se présentent dans notre contexte national comme une nouveauté institutionnelle et donc comme un objet dont l'influence dans le champ politique est pour le moment difficilement identifiable. De ce fait ils méritent l'attention des chercheurs afin d'éclairer aussi bien l'opinion publique que les politiques: ils peuvent a priori être perçus (1) soit comme de simples instruments au service d'un pouvoir néo-libéral facilitant la globalisation de l'économie (comprise comme la nouvelle étape du capitalisme) (2) soit, tel que l'indique le professeur Alain Lancelot, comme des outils en vue de « moderniser et revivifier le débat politique national $»^{1}$. Dans son plaidoyer en faveur des think tanks paru dans la presse, cet ancien administrateur de la Fondation Nationale des Sciences Politiques, insiste à plusieurs reprises, sur le haut niveau d'exigence scientifique des individus œuvrant dans un think tank ainsi que sur leur capacité à maintenir la «bonne » distance entre la recherche pure/fondamentale et l'action. Le think tank répondrait d'après Lancelot à l'impératif d'une réflexion et d'une analyse se situant au dessus des clivages politiques traditionnels (relevant des partis politiques). A priori cette description n'est pas trop éloignée des représentations que se donnent les Américains de leurs think tanks mettant également l'accent en ce début de siècle, sur l'avènement d'une ère post-partisane ${ }^{2}$. Mais précise Lancelot, le « think tank à la française », contrairement à son homologue américain, se garderait bien de jouer de son influence et éviterait en tout état de cause de se comporter comme un lobby intellectuel : «Les think tanks américains se présentent plus comme des lobbies intellectuels engagés qu'à des instances de confrontation et de débat en amont du combat politique ». D'où l'interrogation sur la 
pertinence de cet élément de distinction entre le think tank à la française et le think tank à l'américaine : un think tank menant des travaux de recherche pertinents et un débat de qualité (parce que situé au-delà du débat traditionnel) peut-il se priver de tout pouvoir d'influence?

L'objectif de cet article n'est pas de comparer terme à terme la (possible) figure du think tank français et celle de son homologue américain ancré depuis un siècle dans l'espace politique (plus particulièrement dans la deuxième moitié du $\mathrm{XX}^{\mathrm{e}}$ siècle) dans le but d'en faire un «modèle » à copier ou à rejeter. L'analyse se propose de les replacer dans le contexte politique américain tout en soulignant leur pouvoir d'influence auprès de l'État fédéral. Toutefois loin de porter un regard critique sur leurs capacités d'influence, la réflexion se propose de mettre en évidence les spécificités des représentations sociales et culturelles des Américains à l'égard de l'État dont le rôle est justement perçu comme un arbitre entre les lobbies. L'État est une donnée universelle mais la construction de tout État relève de contingences historiques, ce qui signifie que toute analyse de think tank exige au préalable quelques éléments d'explicitation de la nature des relations think tank-État tout en les contextualisant.

\section{Deux think tanks privilégiant la question urbaine : l'Urban Institute et la Brookings Institution}

La majorité des think tanks américains remonte au milieu du XX $X^{\text {e }}$ siècle même si certains comme la Brookings Institution travaillant sur les relations internationales et sur l'économie date du début du siècle dernier. La Rand Corporation qui en ce début du $\mathrm{XXI}^{\mathrm{e}}$ siècle se situe au premier rang de l'ensemble des think tanks américains pour un budget annuel s'élevant à 251 millions de dollars est certainement la plus connue de l'opinion publique. Elle a pratiquement conceptualisé la politique étrangère de l'État fédéral à l'époque de la guerre froide. Les think tanks sont des institutions qualifiées de «non-profit organizations», l'équivalent de la catégorie «associations » en France. Ils regroupent des experts disposant de capacités d'analyse et de réflexion visant à faire des propositions à l'intention des politiques, propositions susceptibles d'engendrer des politiques publiques pertinentes. On les qualifie alors de «laboratoires d'idées innovantes ». Il est certain que l'avènement de l'administration Reagan a été largement facilité par la révolution conservatrice imaginée, dès les années 1970, au sein de quelques think-tanks. Le think tank est par ailleurs perçu comme une institution autorisant et facilitant la circulation d'une élite intellectuelle, susceptible d'être mobilisée à l'occasion d'un changement d'administration, à l'échelon fédéral.

La plus récente étude sur les think tanks a été menée par le professeur de sciences politiques James McGann, qui va même jusqu'à qualifier certains d'entre eux de "governments in waiting». Directeur-adjoint du programme de recherche Think Tanks and Civil Societies Program à l'Université de Pennsylvanie, McGann a mis au point une typologie dont les trois plus importantes catégories sont : Policy Makers, Scholars et Activists ${ }^{3}$. Les États-Unis se situent au premier rang pour leur nombre de think tanks s'élevant à 1777 pour un chiffre mondial de 5465 . Le deuxième rang est occupé par le Royaume-Uni dont le nombre de 
think tanks s'élève à $283^{4}$.

Le choix de la présente analyse s'est arrêté plus particulièrement sur deux think tanks, la Brookings Institution et l'Urban Institute. Le premier a plus d'un siècle alors que le second date de la deuxième moitié du $X^{\mathrm{e}}$ siècle. Toutefois l'intérêt porté par le premier pour les questions urbaines se situe deux ans après la création du deuxième.

\section{L'Urban Institute et la Brookings Institution}

Pour ce qui concerne la thématique de la question urbaine, les deux think tanks dont les travaux sont les plus diffusés et les mieux connus parmi les chercheurs universitaires et les politiques, sont la Brookings Institution (BI) ${ }^{5}$ et $1^{\text {'Urban }}$ Institute (UI) ${ }^{6}$ souvent qualifiés d'excellents ${ }^{7}$. Ils sont tous les deux localisés à Washington DC et leur origine se situe au milieu de la décennie 60 , sous la présidence de Lyndon B. Johnson. La liste des think tanks travaillant sur la thématique de la ville ne se limite certes pas à ces deux think tanks mais les autres, comme par exemple le Milken Institute localisé à Santa Monica (Californie), le font de manière épisodique ou encore en privilégiant l'angle économique ${ }^{8}$. La Brookings Institution - créée par le philosophe Robert S. Brookings - a déjà un siècle mais le début de son engagement pour la ville est généralement associé au discours du président Johnson à l'occasion des célébrations du $50^{\mathrm{e}}$ anniversaire du think tank, le 29 septembre 1966. La création de l'UI remonte à 1968 : sa genèse correspond à la formulation par une Task Force entourant le président Johnson, de l'impératif de disposer d'un centre de recherches indépendant sur la ville et ses habitants. À cette époque, l'influence de la célèbre École de sociologie qualifiée d'École de Chicago étaient perçue comme peu pertinente pour deux raisons : ses travaux ne prenaient pas en compte les dimensions raciales et ethniques de la société américaine et ils ne s'appuyaient pas sur des données statistiques. Pour les politiques comme pour l'opinion publique, il devenait de plus en plus difficile de dissocier les problèmes sociaux, l'accès à l'enseignement public et la pauvreté en dehors de données statistiques prenant en compte la question raciale. Aussi il revenait à ces deux centres (BI et UI notamment) de mieux connaître les besoins de la population vivant en dessous du seuil de la pauvreté et de contribuer au débat en faveur de l'égalité des chances des Américains au-delà de leur appartenance raciale et ethnique. Une très grande importance avait été ainsi accordée à l'amélioration et à la qualité de l'offre des services publics dans les ghettos, un terme de moins en moins utilisé en ce début du $\mathrm{XXI}^{\mathrm{e}}$ siècle en raison des profondes recompositions et reconfigurations sociales, économiques et politiques des territoires urbains. En simplifiant un peu, on peut dire que les études de l'Urban Institute abordent de front les questions sociales et les programmes éducatifs alors que le BI privilégie le registre économique.

La création de ces deux centres correspond à un moment où l'État fédéral - probablement sous l'influence des maires des grandes villes - souhaite s'intéresser aux villes. À cette époque, les universitaires travaillaient certes sur la ville mais avaient tendance à se limiter aux villes des États où l'université était localisée. Par ailleurs, on disposait de peu de travaux empiriques menés de manière systématique sur l'ensemble des grandes villes et leurs populations. La décennie 60 coïn- 
cide également avec l'émergence de méthodes de travail et d'outils informatiques tirant avantage de la richesse des données statistiques du Bureau du recensement. Les chercheurs des think tanks furent les premiers à s'en servir. Toutes les conditions étaient donc réunies pour donner à l'État fédéral, une représentation fiable et cohérente de la question urbaine sur l'ensemble de son territoire. L'État fédéral s'approprie la thématique du registre urbain et a des moyens de comparaison entre les villes.

\section{Des études en complémentarité avec la recherche universitaire}

Le professeur McGann situe la BI dans la catégorie de Scholars (chercheurs hautement spécialisés) et l'UI dans celle de Policy Makers. Le second ayant donc une influence directe sur la conception des politiques publiques. Cette distinction paraît toutefois peu pertinente si l'on observe les récentes initiatives du président Obama concernant la création d'un Bureau des Politiques Urbaines à la Maison Blanche : la BI peut être qualifiée de Policy Makers. En revanche, le changement dans le statut des chercheurs s'avère intéressant à souligner. Au moment de leur création, ces deux think tanks disposaient d'une équipe de chercheurs à plein temps. En ce début de $\mathrm{XXI}^{\mathrm{e}}$ siècle, la $\mathrm{BI}$ a opté pour plus de flexibilité dans la gestion de la recherche, ce qui lui permet également d'étendre son réseau d'influence : outre ses chercheurs à plein temps, elle inclut des chercheurs qui ont le statut de professeurs affiliés à des universités. Ces derniers sont qualifiés de « non resident fellow ». Le programme de la BI intitulé Metropolitan program compte ainsi deux éminents professeurs, Myron
Orfield et Robert Lang, le premier de 1'Université du Minnesota à Minneapolis/Saint-Paul et le second de l'Université de Virginia Tech à Alexandria (Virginie). On ne retrouve pas pour le moment l'équivalent de ce statut pour l'UI. La BI et l'UI, deux institutions de qualité, exercent une influence certaine dans le champ politique mais les Américains différencient l'influence de l'activisme. L'attitude d'une think tanks comme la Human Rights Watch dont l'objectif est la défense des droits de l'homme dans le monde, se rapproche plus du prosélytisme que de l'influence à proprement parler.

Ce rappel sur la création de deux think tanks (localisés à WDC) ainsi que leur part d'influence dans l'espace politique, permet d'emblée de préciser qu'ils sont loin de se situer dans un positionnement de rivalité avec des départements de sciences sociales d'universités (prestigieuses ou non). La plupart des universités américaines ont commencé à se doter de programmes ou de départements d'études urbaines au début des années 1970, après la création de ces deux think tanks. La réflexion menée au sein d'un think tank est conduite avec pragmatisme et avec un souci permanent de contribuer à la formulation de politiques publiques efficaces et performantes. Elle repose, en outre souvent sur des chercheurs travaillant en équipe et sur le mode de la transdisciplinarité, ce qui explique en grande partie leurs capacités d'influence sur les politiques. Comme le soulignent les débats en cours sur la relance économique au travers de l'investissement public dans les réseaux techniques, les think tanks sont en mesure de fournir des données et des arguments associant les qualifications des ingénieurs, des économistes et des financiers. 
Aux États-Unis les think tanks ne sont pas vécu comme une menace pour les institutions relevant de l'enseignement supérieur ou de la recherche dans la mesure où ils se présentent comme des lieux menant des recherches finalisées ou encore tournées vers l'action publique réservant ainsi la recherche fondamentale aux universités. Les travaux menés par les think tanks présentent par ailleurs l'avantage de faire l'objet d'une vaste diffusion loin d'être limitée aux seuls spécialistes de la question. Au cours de ces 15 dernières années, les think tanks ont mis leurs rapports d'études en ligne dès leur parution et certains d'entre eux sont repris par la presse, ce qui permet de contribuer à la formation de l'opinion publique. Les think tanks, se retrouvent ainsi dans une situation de complémentarité avec les universités, s'appuient sur des nouveaux outils informatiques et comblent un vide dans la production des connaissances au service de la décision publique.

Rendre compte de l'influence des think tanks sur la politique de l'État fédéral s'appuie ici sur la conférence donnée par le directeur du programme urbain de la BI, Bruce Katz, à l'Université de New York en février 2009. Quant à la note du mois de mars 2009, intitulée «ARRA : An early assessment of the American Recovery and Reinvestment Act», elle indique que le think tank n'hésite pas à faire preuve d'autorité critique à l'égard de l'État fédéral quand ce dernier modifie sa ligne de conduite.

\section{Les cent premiers jours de l'administration} Obama au travers du prisme des think tanks

L'influence du think tank BI sur la politique menée par le président Obama a été affichée de manière explicite dans le cadre d'une conférence publique donnée à l'université de New York le 2 février 2009. Bruce Katz a commencé son allocution en disant qu'il avait fait partie de l'équipe de transition nommée par le président au lendemain de son élection (novembre 2008) et qu'il était à ce jour « conseiller » du ministre du Logement et du Développement urbain (Secretary of housing and urban development), Shawn Donovan. Sa conférence avait en fait pour objectif d'expliquer au public les missions et objectifs du « Bureau des politiques urbaines », une instance créée à la Maison Blanche par le président Obama.

\section{La création d'un «Bureau de politiques urbaines » à la Maison Blanche}

Bruce Katz a rendu compte de la légitimité et de la pertinence de la création d'un Bureau centré sur les questions urbaines à la Maison Blanche en reprenant pour l'essentiel des arguments construits au cours des dernières années dans les nombreux travaux publiés sur le sujet. Il a indiqué dans un premier temps combien à l'heure des flux d'échanges globaux (finance, information, connaissances), la thématique des territoires devenait une priorité et combien les politiques publiques de l'État fédéral se devaient de contribuer à renforcer l'attractivité des territoires et des lieux'. Il a ensuite déclaré tout en l'explicitant combien la dynamique économique s'avérait indissociable de la dynamique des entités urbaines et métropolitaines. Le positionnement des villes ou encore villes-régions ${ }^{10}$ américaines dans les flux globaux devenait désormais un enjeu national et qu'il revenait à l'État fédéral de s'en préoccuper. Dans le cadre de cette conférence publique, Bruce Katz a pris le rôle du chercheur expliquant l'impératif 
d'une « nouvelle » représentation de l'économie américaine ne se limitant plus à la somme des économies générées par les 50 États fédérés plus ciblées sur les villes. Mais il a également démontré combien l'initiative du président Obama correspondait bien à ce nouvel impératif visant à faire des villes et aires urbaines, les locomotives d'une relance économique sous la responsabilité de l'État fédéral. Katz a ainsi précisé combien l'État fédéral se devait de répondre au défi de la perspective du développement durable (à l'heure du changement climatique).

L'avènement de régions urbaines ( $«$ sustainable urban regions ») exige désormais de concevoir des politiques publiques en mesure d'inclure les thématiques du logement, du transport, de l'énergie et de l'aménagement urbain. Le rôle de l'État fédéral n'est pas d'encourager la construction de maisons et bâtiments à faible dépendance énergétique ou encore de ne financer la construction de logements qu'à la condition qu'ils soient reliés à des transports en commun mais de s'assurer qu'à l'échelon local, s'instaure et se crée une convergence entre les politiques publiques sectorielles menées par l'échelon fédéral. Katz va encore plus loin dans son argumentation visant à expliquer et à légitimer la création d'un Bureau de politiques urbaines au sein de la Maison Blanche en évoquant le principe d'un fédéralisme américain non figé. Il souligne ainsi combien les Pères fondateurs se sont gardés de définir avec précision les modalités de fonctionnement du fédéralisme afin de permettre à la vie politique de s'adapter aux exigences du contexte historique. La présentation de Katz inclut également quelques citations du président Obama en particulier celles de son discours de campagne présidentielle en juin 2008 à
Miami. Obama rappelait alors que l'État fédéral se devait d'accorder une priorité aux villes (et métropoles) qui font face à des mutations (sociales et économiques) majeures et qui représentent des lieux privilégiés de la vie économique. Le candidat Obama avait par ailleurs souligné que la croissance démographique ne concernait pas uniquement les villes mais également les banlieues ainsi que le périurbain : " la Silicon Valley ne se limite pas à la seule municipalité de Palo Alto où sont localisées l'université Stanford et les principales entreprises innovantes mais elle inclut également d'autres municipalités voisines ».

Katz reprend également des données issues des travaux de sa recherche. Les 100 premières aires métropolitaines possèdent plus des $2 / 3$ du nombre total d'emplois, génèrent $80 \%$ des brevets et assurent $75 \%$ des échanges commerciaux et 42 aires métropolitaines américaines se retrouvent dans la liste des 100 premières économies mondiales. D'où l'importance que l'État fédéral doit leur accorder pour mener à bien une politique économique stratégique alors que jusqu'ici Washington a vu son rôle limité à la prise en charge des problèmes sociaux de la ville sans se soucier bien entendu des aires métropolitaines. Katz rappelle également combien le candidat Obama est sensible à la question urbaine en raison de son expertise en tant que « community organizer » à South Park (ghetto noir de Chicago). Il faut certes combattre la pauvreté, la criminalité et la violence mais il faut également construire des régions urbaines fortes afin de construire une Amérique forte.

La conférence de Katz à l'Université de NY est certes intéressante pour toute personne s'intéres- 
sant à la ville et à la question urbaine dans la mesure où elle synthétise de manière concentrée et pertinente l'essentiel des résultats du programme de travail de la $\mathrm{BB}$. Mais au-delà de ces références aux travaux réalisés et publiés, la conférence de Katz indique clairement l'influence du think tank sur la stratégie économique et urbaine du président Obama. En mettant en évidence l'originalité du parcours d'Obama qui figure désormais comme le premier président américain à avoir eu une expérience de terrain (professionnelle et politique) en milieu urbain, tout lecteur est en mesure de déduire l'influence du tt sur le président pour ce qui concerne les enjeux économiques et politiques des aires métropolitaines ${ }^{11}$. Pour Katz, le rôle de l'État fédéral n'est plus de distribuer des fonds aux États (notamment pour les infrastructures) mais d'élaborer des normes et exigences afin que ces réseaux techniques contribuent à la structuration d'un espace territorial et non à l'étalement à l'infini du tissu urbain. Pour éviter de se conformer aux modalités traditionnelles des transferts financiers de l'État fédéral en direction des juridictions territoriales, le Bureau des politiques urbaines de la Maison Blanche aura pour mission de veiller à la « cohérence spatiale » du programme de relance économique.

\section{Le think tank : entre pouvoir d'influence et pouvoir critique}

La conférence du 2 février 2009 confirme l'hypothèse d'une influence certaine du think tank sur la politique de l'État fédéral ainsi que sur la campagne présidentielle. Par ailleurs l'annonce du programme d'études comme par exemple la construction d'indicateurs pour la répartition des fonds fédéraux entre les cent premières aires métropolitaines (Metropolitan Investment Review), précise le travail d'accompagnement que se propose de faire la BI pour assurer la soutenabilité/ durabilité (soit la prise en compte du moyen terme) des aires métropolitaines et ainsi affirmer le principe d'un régionalisme centré sur la ville ${ }^{12}$. Le think tank n'hésite pas pour autant à faire preuve de positionnement critique lorsque l'État fédéral n'agit pas de manière conforme aux principes avancés.

La BI a diffusé en mars 2009, sur son site Internet une note "Metro potential in ARRA : An early assessment of the American Recovery and Reinvestment Act » qui, comme son nom l'indique, se veut une évaluation ex-ante de la loi pour la relance de l'économie américaine votée en février 2009 par le Congrès. Le document commence par affirmer que la « crise économique » américaine est avant tout une « crise métropolitaine » parce que désormais l'économie américaine ne se veut plus la somme de l'économie de 50 États, mais relève plutôt de la vitalité des aires métropolitaines. Puis il n'hésite pas à porter un regard plutôt critique vis-à-vis de la loi en faveur de la relance économique (votée par le Congrès) parce que celle-ci risque de se limiter à des flux financiers en direction des États (et parfois des municipalités) suivant des critères traditionnels ne prenant donc pas en compte la cohérence métropolitaine. Or indique la note, les États fédérés n'ont pas encore pris conscience de la dynamique économique de leurs métropoles et de ce fait sont susceptibles de distribuer la manne fédérale aux élus et territoires en fonction de leur proximité politique. La note ne se veut pas uniquement un document critique, elle va plus loin et encourage les acteurs locaux (publics ou privés) et l'opinion 
publique à militer pour faire pression auprès des États fédérés pour que les dépenses d'infrastructures (fonds fédéraux) assurent et renforcent la cohérence métropolitaine. La quatrième partie de la note intitulée "Metro Governance » met l'accent sur l'impératif de conceptualiser une politique d'infrastructures (routes, autoroutes, énergie, eau) à l'échelle métropolitaine, parallèlement aux aides pour le logement. Elle s'appuie sur travaux d'économistes comme Michael Porter ou Anna Lee Saxenian affirmant que désormais l'« avantage comparatif » à l'heure des flux se joue à l'échelle des aires métropolitaines.

La conclusion du rapport/note affirme que si les financements fédéraux actuels ne sont pas vraiment pensés pour répondre aux attentes des habitants et des acteurs économiques des métropoles, il revient alors aux États fédérés, acteurs locaux et à l'opinion publique de faire preuve d'innovation et de s'assurer que les fonds fédéraux sont dépensés de manière à assurer la cohérence territoriale de l'aire métropolitaine. Le think tank donne son accord à la politique d'investissement public mené par l'État fédéral mais en même temps il sait prendre ses distances par rapport à l'État fédéral sous prétexte qu'il lui revient de s'assurer de la performance des fonds publics et des politiques publiques. L'idée de la maximisation des investissements publics par une prise en compte des aires métropolitaines est ainsi récurrente dans l'analyse.

\section{Conclusion : le think tank et les représen- tations culturelles de l'État}

Les think tanks jouent un rôle considérable dans la vie politique américaine. Ils ont un pouvoir d'influence sur la conceptualisation des politiques publiques tout en évitant de concurrencer les universités et leurs centres de recherche. Les programmes de recherche des deux think tanks (BI et UI) correspondent au souci de l'État fédéral qui, dès le milieu des années 1960, se propose d'inclure dans sa politique les villes. Ce contexte politique résulte en fait de l'étonnement du candidat à l'élection présidentielle de l'époque, John F. Kennedy qui à l'occasion de sa campagne, découvre l'existence de ghettos raciaux et la misère de sa population. L'État fédéral reconnaît qu'il ne possède aucune expertise sur ce sujet dans la mesure où les villes ne figurent pas dans la Constitution, et qu'il a besoin d'une expertise indépendante et pertinente pour l'ensemble de son territoire.

Pour ce qui concerne la politique de relance économique menée par le président Obama, l'analyse a démontré le poids incontournable de la BI au travers de son programme Metropolitan program. La BI, représente le lieu institutionnel de la formation de l'idée d'un partenariat entre l'État fédéral, l'État fédéré, les gouvernements locaux et métropolitains et le lieu par excellence où le principe d'une coordination entre les politiques sectorielles (concernant l'énergie, le changement climatique et les transport) a été le mieux formalisé. Le think tank se propose donc non seulement d'améliorer la performance des politiques publiques fédérales par un travail rigoureux mais également se propose d'être le lieu où se pensent les réformes progressives visant à renouveler l'action publique. L'influence du think tank sur le politique et l'opinion publique est incontournable.

Tout en reconnaissant le pouvoir d'influence du 
think tank dans l'espace politique américain, il est possible de conclure que ce pouvoir ne représente pas un défi pour le fonctionnement de la vie politique américaine. En effet aux États-Unis, l'État n'est pas perçu comme un lieu où se fabrique l'intérêt général et où se défend l'idée d'une égalité entre les citoyens. L'État est tout simplement perçu comme une institution en mesure de maintenir l'unité entre les 50 États fédérés et comme une institution dont le rôle majeur est de se situer en arbitre permanent entre les différents courants et entre les multiples lobbies politiques. Dans ce cadre, le think tanks se perçoit alors comme un lobby professionnel parmi d'autres. Il est donc difficile de concevoir un tt d'excellence dénué de tout pouvoir d'influence.
$\mathrm{N} \cdot \mathrm{O} \cdot \mathrm{T} \cdot \mathrm{E} \cdot \mathrm{S}$

1. Alain Lancelot, « $\mathrm{La}$ république a besoin de savants ", Le Figaro, 3 novembre 2003. L'auteur utilise de préférence le terme de «fondation » mais en fait il s'agit de « think-tank ».

2. L'expression «post-partisan politics» a été largement utilisée par les médias américains dans la seconde phase de la campagne présidentielle de 2008, au moment où le candidat démocrate affrontait le candidat républicain.

3. James McGann, The Think Tank Index, University of Pennsylvania, 2008.

4. En France, le nombre de think tanks se situe autour de 165 .

5. Brookings Institution basé à Washington D.C. est un organisme non-lucratif et indépendant dont la mission est de mener des recherches en vue de formuler des politiques publiques. Ses recommandations innovantes et pragmatiques se proposent de poursuivre trois objectifs : renforcer la démocratie américaine, encourager le bien-être économique et social, la sécurité et l'égalité des chances pour tous les Américains, et enfin promouvoir un système de coopération internationale plus sécurisé, plus ouvert et plus prospère. Comme l'indique de manière explicite son site Internet, Brookings Institution est fière d'être citée et considérée comme un think tank de grande qualité et doté d'un réel pouvoir d'influence. Ses travaux relèvent de cinq programmes: Économie, Gouvernance, Politique Étrangère, Programme Métropolitain et Économie Globale.

6. Urban Institute-lequel rassemble des données et des statistiques, conduit des recherches et évalue des programmes - se donne pour mission d'éduquer les Américains sur les enjeux sociaux et politiques majeurs, et cela afin de renforcer les politiques publiques de qualité 
et de rendre le gouvernement plus performant. Une des spécificités de l'Urban Institute - l'un de ses principaux atouts - repose sur l'usage de la modélisation mathématique et de techniques statistiques performantes lui permettant d'évaluer les politiques publiques mais également de simuler les comportements des ménages. Cette expertise l'autorise en outre à anticiper sur les impacts (possibles) des politiques publiques à l'égard notamment des ménages pauvres. Ses capacités et compétences techniques lui permettent enfin de jouer le rôle de « conseiller technique » auprès de diverses organisations à l'étranger.

7. Les travaux de ces deux think tanks s'appuient sur les statistiques démographiques, sociales et économiques du Bureau du recensement, un organisme fédéral dont les travaux sont hautement respectés par les chercheurs, les politiques, les médias et l'opinion publique. Aussi, l'Urban Institute a-t-il mené sur la base du résultat des recensements entre 1970 et 2000 une étude sur les changements socio-économiques et démographiques des quartiers des grandes villes américaines (Neighborhood Change Database).

8. Consulter l'ouvrage The Best Performing Cities index: Where America's jobs are created and sustained, 2008 qui en fait se limite, comme l'indique le titre, à hiérarchiser les villes en fonction de leurs capacités à créer et maintenir des emplois.

9. La notion de lieu est une traduction du terme «place» utilisé par les Américains. Au cours de la dernière décennie, de nombreux géographes et sociologues ont eu l'occasion de s'exprimer sur ce sujet. Voir notamment l'ouvrage de Dreier P., Mollenkopf J. \& Swanstrom T., Place Matters : Metropolitics of the 21st Century.

10. Katz parle d'«aires métropolitaines», ce qui est en fait synonyme de l'expression « city-region » également utilisée par les chercheurs américains.

11. Cynthia Ghorra-Gobin, «Barack Obama, 44 président des États-Unis: événement historique ou simple figure symbolique? », Quaderni, $\mathrm{n}^{\circ}$ 68, pp. 113-123.

12. Le débat sur le régionalisme n'est pas récent. Il a connu une brève période dans les années 1960 et a réémergé il y a environ une décennie, parallèlement à la prise de conscience de l'impact de l'avènement de l'économie globale sur les villes. On lira à ce sujet $\mathrm{C}$. Ghorra-Gobin, Les États-Unis entre local et global, Presses de Sciences Po, 2000. 
$R \cdot E ́ \cdot S \cdot U \cdot M \cdot E ́$

Cet article se propose d'éclairer le récent débat francophone sur la place et le rôle du think tank dans le discours social à partir d'une analyse ayant pour objet deux think tanks américains, la Brookings Institution et l'Urban Institute. Il met en évidence la stratégie du think tank optant pour un positionnement de complémentarité avec les centres de recherches des universités. Il souligne également que compte tenu de la qualité de ses travaux, le think tank ne se prive pas de jouer de son influence notamment auprès de l'État fédéral, comme l'atteste la période de transition de l'administration Obama au début de l'année 2009.

The purpose of this article is to enlighten the current francophone debate about the role of think tanks in social discourse, based on an analysis of two American think tanks, Brookings Institution and Urban Institute. It underlines the strategy of think tanks for reaching a certain complementarity with university research centers. It also stresses that given the quality of their work think tanks do not hesitate about using their clout and thus influence the Federal government as shown by the transition period of the Obama administration in the beginning of 2009 . 
